\title{
ANÁLISIS DE LA COMPETITIVIDAD DE LA NARANJA ESPAÑOLA A NIVEL MUNDIAL
} (PÓSTER)

\author{
$\underline{\text { R. Ben Amor }}{ }^{1}$ y MD. De-Miguel Gómez ${ }^{1}$
}

${ }^{1}$ Departamento de Economía de la Empresa, ETSIA, Universidad Politécnica de Cartagena, España. Resumen

A nivel mundial, España ocupa el sexto puesto como productor de cítricos, con una citricultura especializada para el consumo en fresco. Las naranjas representaron el 52,1\% de la producción total citrícola española en la campaña 2018/19, seguidas de las mandarinas, con un 31,9\%, los limones con $15 \%$ y pomelos y otros con $1 \%$. El cultivo y la producción de naranja tienen una larga tradición y clara importancia estratégica en términos de desarrollo económico, social y ambiental, siendo las principales zonas productoras: la Comunidad Valenciana, Murcia, Andalucía y Cataluña.

La ventaja comercial que ha tenido las naranjas españolas se ha visto afectada por la globalización de los mercados provocando una grave crisis en la citricultura española, lo que ha repercutido en la producción y exportación de naranjas, ello obliga a realizar un análisis de la situación, utilizando los principales indicadores de competitividad. El análisis de la ventaja competitiva de la naranja española a nivel mundial, durante las últimas 24 campañas, refleja una tendencia positiva del sector, pero presenta indicios de acercamiento a los límites de competitividad, manteniendo el liderazgo como principal país exportador de naranjas a la UE, lo que coloca a España por delante de sus competidores tradicionales.

Palabras clave: España, mundo, índices de competitividad, exportaciones, cítricos.

\section{Introducción}

Actualmente, el comercio de frutas y hortalizas se encuentra en una situación de alta competitividad internacional y la globalización ha hecho que las empresas de todo el mundo sean más competitivas. Esta competitividad depende del principio de ventajas comparativas, asociadas con factores naturales favorables y con menores costes de producción (Fidan, 2009), y que puede explicar la especialización de un país en el comercio internacional, si no hubiera distorsiones en los mercados.

España, es el sexto productor mundial de cítricos y naranjas, con un 5,6\% y un 4,3\% respectivamente de la producción mundial en 2016 (FAO, 2018), y es el mayor productor de cítricos para consumo en fresco. Las naranjas representan el 53\% de la producción total española en la campaña de 2017/18 (3.357.163tn), seguidas de las mandarinas (31\%) y los limones (15\%) (MAPA, 2019). La producción de cítricos en España se encuentra principalmente en cuatro regiones: Comunidad Valenciana, Murcia, Andalucía y Cataluña. España es el primer exportador de cítricos y naranjas en el mundo en los últimos 20 años, con un 24,5\% y un $23,6 \%$ respectivamente de exportaciones mundiales (FAO, 2018).

Los principales países productores y exportadores de naranjas en el mundo, competidores directos de España, son: Egipto, Sudáfrica y Marruecos y si incluimos a España, queda garantizado el $91 \%$ de la producción mundial de naranja, de los cuales el 22,4\% corresponde a España.

Por lo que este trabajo propone estudiar la posición competitiva de un sector estratégico para la economía de España como es el sector citrícola, especialmente la naranja, frente a sus principales competidores, proporcionando un análisis evolutivo en el mundo de los principales indicadores del intercambio comercial de exportación e importaciones de las naranjas, como son la ventaja comparativa revelada y la competitividad del mercado (y su evolución temporal). A partir de este estudio, establecemos el perfil de exportación de diferentes países como: España, Egipto, Sudáfrica, Marruecos, Turquía, Brasil, EEUU, Argentina y Túnez.

\section{Material y Métodos}

\section{1. Índices de competitividad económica}

\subsection{1. Índice de ventaja comparada revelada de Balassa (IVCR)}

Balassa (1965) la definió como la relación entre las exportaciones de ciertos productos de un país y las exportaciones totales de este país al mundo. El Índice de ventaja comparativa revelada (IVCR) se define como:

$$
\operatorname{IVCR}_{\mathrm{ij}} \text { o ISB } \mathrm{ij}=\left[\frac{\left({ }^{x_{i j}} / \sum x_{j}\right)}{\left(X_{j} / \sum X_{j}\right)}\right] \times 100
$$

- IVCRij o ISBij Representa la ventaja comparada revelada de un país i para un producto j

- $\boldsymbol{x}_{\boldsymbol{i} j}$ Exportaciones de un país i para un producto j. 
- $\quad \sum \boldsymbol{x}_{\boldsymbol{j}}$ Exportaciones de todos los productos de un país i.

- $\boldsymbol{X}_{\boldsymbol{j}}$ Exportaciones de un producto $\mathrm{j}$ en la zona de referencia

- $\sum \boldsymbol{X}_{\boldsymbol{j}}$ Exportaciones de todos los productos de la zona de referencia.

Este índice varia ente 0 y $+\infty$, valores inferiores a 100 (200) indican que el país i es relativamente menos especializado en el sector $\mathrm{j}$ dentro del área de referencia, o representa una desventaja en la zona de referencia.

\subsection{2. Índice de dependencia (ID)}

Se define como la relación entre las importaciones de ese sector con respeto a las importaciones totales, ponderando esta relación con el cociente entre las importaciones de ese mismo sector y las importaciones totales de la zona de referencia.

$$
\mathrm{ID}_{\mathrm{ij}}=\frac{\left(m_{i j} / \sum m_{j}\right)}{\left(M_{j} / \sum M_{j}\right)} \times 100
$$

- IDij Es el índice de dependencia de las importaciones del sector i de un país j

- $\boldsymbol{m}_{\boldsymbol{i j}}$ Importaciones de un país i para un producto j.

- $\sum \boldsymbol{m}_{\boldsymbol{j}}$ Importaciones de todos los productos de un país i.

- $\quad \boldsymbol{M}_{\boldsymbol{j}}$ Importaciones de un producto j en la zona de referencia

- $\quad \sum \boldsymbol{M}_{\boldsymbol{j}}$ Importaciones de todos los productos de la zona de referencia

Un índice superior a 100 señala que el país i es más dependiente en la importación del producto j que la zona de referencia.

\subsubsection{Cuota de mercado constante (CMS)}

Es una técnica que analiza los patrones de comercio y las tendencias con el fin de formular políticas. Desagrega los datos del comercio de los países estudiados y los compara con los flujos comerciales del resto del mundo. La ecuación tradicional del CMS es:

$$
\mathrm{CMS}_{\mathrm{ij}} \circ \mathrm{PM}_{\mathrm{ij}}=\left[\frac{X_{i j}}{X_{m}}\right] \times 100
$$

- $\quad \mathbf{C M S}_{\mathrm{ij}} \mathbf{0} \mathbf{P M}_{\mathrm{ij}}$ Cuota de mercado constante de un producto $i$ de un país $j$

- $\quad \boldsymbol{X}_{i j}$ Exportaciones de un país $j$ de un producto $i$.

- $\boldsymbol{X}_{m}$ Exportaciones de la zona geográfica de referencia de un producto $i$.

\subsection{4. Índice de Balanza Comercial (TBI)}

Definido como la relación entre la exportación y la producción total de bienes objeto de comercio. El TBI explica si un país es un exportador neto o un importador neto. Para simplificar la interpretación del índice TBI, señalamos si el índice es positivo significa que es un país exportador neto y cuando el índice TBI es negativo se habla de que el país es importador neto. Se formula de la siguiente forma:

$$
\mathrm{TBI}_{\mathrm{ij}}=\left[\left(\frac{x_{i j}-\mathbf{M}_{i j}}{x_{i j}+\mathbf{M}_{i j}}\right)\right] \times 100
$$

- $\quad$ TBI $_{\mathbf{i j}}$ Es el índice de balanza comercial de un país $i$ para un producto $j$.

- $\quad \boldsymbol{X}_{i j}$, Exportaciones del país $i$ de un producto $j$.

- $\quad \mathbf{M}_{i j}$, Importaciones del país $i$ de un producto $j$.

El valor del índice TBI varía entre -100 y 100. Cuando TBI está próximo a 100, indica que la estructura cualitativa de las exportaciones para un país $i$ está por encima de la estructura de sus importaciones, o se habla de un país exportador neto. Al contrario, si TBI es igual o cerca de -100, implica que el país $i$ es importador neto. Si el valor del índice TBI es igual o cerca de cero, señala que el valor de las exportaciones es igual al valor de las importaciones del país $i$.

\subsection{Recopilación de datos}

El estudio se realizó de los principales países productores/consumidores de la naranja en el mundo. Las estadísticas del comercio agrícola y de las naranjas, fueron tomadas de la FAO (2019). 


\section{Resultados y discusión}

La tabla 1 muestra que, España, durante las 24 campañas del periodo estudiado, desde 1992 a 2016, por término medio, ha pasado de ocupar el $2^{\circ}$ puesto al $4^{\circ}$, en cuanto al VCR o índice de especialización, es el valor más alto para las naranjas, con una media de 1.630,07 (muy superior a 100) que se ha mantenido estable a lo largo del periodo de estudio.

La participación española en la estructura de la exportación es relevante, con una media de 96,20 (ID inferior pero muy cercano a 100), con lo cual se puede decir que España está fuertemente especializado en este sector, aunque el índice de dependencia muestra que España es cada vez más dependiente de las importaciones de naranja para poder satisfacer la demanda exterior de este cítrico y mantener un VCR alto y estable.

La estabilidad en el mercado mundial que tiene España queda reflejada en la cuota de mercado, CMS, que a lo largo del periodo solo sufrido ligeras variaciones. Los valores de CMS, muestran que 29,16\%, de media de las exportaciones mundiales de naranjas corresponden a España.

Los valores de TBI, oscilan entre 96,5 y 84,8, con un ligero descenso, sus valores son positivos y muy cercanos a 100, lo que indica que la estructura cualitativa de las exportaciones españolas está por encima de la estructura de sus importaciones, es decir, se clasifica como un exportador neto, para el sector del naranjo.

Egipto, Marruecos y Sudáfrica, son los principales competidores de España en el sector de los naranjos. Egipto ocupa el primer puesto, con un VCR medio de 3.260,50, valor que se ha multiplicado por 6, debido al fuerte aumento de sus exportaciones, sector que ha mejorado su producción y respondiendo a la alta demanda del mercado exterior. Su participación en el mercado CMS ha alcanzado el valor de 9,9\%, compitiendo con los principales exportadores de naranjas en el mundo. El ID de Egipto presenta una media de 0,5 (casi igual a 0), señalando que Egipto importa cantidades insignificantes o no importa naranjas, es un país no dependiente de las importaciones de naranjas en el mundo, lo que confirma su TBI $\approx 100$, y se clasifica como exportador neto.

Marruecos, es el $2^{\circ}$ más especializado, con un VCR medio de 3.121,52 (tabla 1) superando 2 veces a España, aunque se ha visto bastante afectado, perdiendo una gran parte de cuota de sus exportaciones, lo que confirma los valores de su participación en el mercado (CMS), que ha pasado de 5,2 a 1,4. El ID de Marruecos es 0 indicando que sus importaciones son nulas, debido a esto, su TBI es de una media de 100, y se clasifica como exportador neto.

El tercer lugar lo ocupa Sudáfrica, se clasifica como un país especializado en naranjas a nivel mundial, su VCR es de 1.724,73, duplicándose entre el primer y el último tramo de estudio, mejorando su participación el mercado (CMS) que de 6,3 ha pasado a 12,5, presentando un aumento continuo y progresivo. El ID de Sudáfrica, con una media de 3,4 indica que en cada tramo las importaciones de naranjas han ido en aumento, lo que confirma su participación en el mercado, mantiene una media estable de 99,6, que le clasifica como exportador neto.

Con VCR mucho más bajos se encuentran Turquía, Túnez, Argentina y USA (EEUU), con valores superiores a 100, indicando que son países especializados en el sector de naranjas. Turquía mejora su posición a lo largo del periodo de estudio. Sin embargo, Túnez y Argentina se ven afectadas con descensos continuos de sus índices.

\section{4.- Conclusiones}

El sector citrícola en España es importante, y concretamente sus naranjas, en términos de producción y exportación, desempeñando un papel socioeconómico clave. Dado el análisis de la competitividad de este sector, concluimos que España tiene una posición comercial muy importante en comparación con el resto de los países del mundo. No obstante, se enfrenta a una fuerte competitividad en los países de la zona, como Marruecos, Egipto y Sudáfrica, que son productores y exportadores fuertes, pero no importadores de estas frutas.

Sin embargo, los índices de participación de mercado para España, son más fuertes y mucho más estables que los principales países competidores. Esto indica que mantiene su mercado a nivel mundial y está muy por encima de todos los países competidores durante el período analizado. Esto resalta la importancia de la naranja española en el mundo. Por otra parte, el índice de la balanza comercial española se mantiene estable, y cerca de 100 para las 24 campañas analizadas, lo que refleja que sigue siendo un exportador neto de naranjas, con una importante estructura de importaciones, caso contrario a lo que ocurre con sus países competidores. 


\section{Referencias bibliográficas}

Balassa, B. (1965). Trade liberalisation and 'revealed' comparative advantage. The Manchester School of Economics and Social Studies 33, 99-123

FAO (Food and Agricultural Organization) (2018). www.fao.org

Fidan, H. (2009). Comparison of Citrus Sector Competitiveness between Turkey and EU-15 Member Countries. HORTSCIENCE. 44(1):89-93.

MAPA, (2019). Anuario de estadística agraria. Ministerio de Agricultura, Pesca y Alimentación.

\begin{tabular}{|c|c|c|c|c|c|c|c|c|c|c|c|c|c|c|c|c|c|c|c|c|}
\hline \multirow[b]{3}{*}{ PAISES } & \multicolumn{20}{|c|}{ Tabla 1.- Análisis de la competitividad no-precio de la naranja española en el mercado internacional } \\
\hline & \multicolumn{5}{|c|}{ VCR } & \multicolumn{5}{|c|}{ ID } & \multicolumn{5}{|c|}{ CMS } & \multicolumn{5}{|c|}{ TBI } \\
\hline & $\begin{array}{c}1992- \\
1996 \\
\end{array}$ & $\begin{array}{l}1997- \\
2001\end{array}$ & $\begin{array}{l}2002- \\
2006\end{array}$ & $\begin{array}{l}2007- \\
2011\end{array}$ & $\begin{array}{l}2012- \\
2016\end{array}$ & \begin{tabular}{|c|}
$1992-$ \\
1996 \\
\end{tabular} & $\begin{array}{l}1997- \\
2001\end{array}$ & \begin{tabular}{|l|}
$2002-$ \\
2006 \\
\end{tabular} & \begin{tabular}{|l|}
$2007-$ \\
2011 \\
\end{tabular} & \begin{tabular}{|l|}
$2012-$ \\
2016 \\
\end{tabular} & \begin{tabular}{|c|}
$1992-$ \\
1996 \\
\end{tabular} & \begin{tabular}{|l|}
$1997-$ \\
2001
\end{tabular} & \begin{tabular}{|c|}
$2002-$ \\
2006 \\
\end{tabular} & \begin{tabular}{|l|}
$2007-$ \\
2011 \\
\end{tabular} & \begin{tabular}{|l|}
$2012-$ \\
2016 \\
\end{tabular} & $\begin{array}{l}1992- \\
1996\end{array}$ & \begin{tabular}{|l|}
$1997-$ \\
2001 \\
\end{tabular} & \begin{tabular}{|c|}
$2002-$ \\
2006 \\
\end{tabular} & \begin{tabular}{|l|}
$2007-$ \\
2011 \\
\end{tabular} & \begin{tabular}{|l|}
2012 \\
2016 \\
\end{tabular} \\
\hline China & 30,93 & 39,83 & 22,95 & 24,48 & 27,97 & 294,3 & 308,0 & 130,7 & 69,8 & 78,5 & 0,8 & 1,4 & 1,4 & 2,4 & 3,5 & $-79,6$ & $-70,7$ & $-62,5$ & $-36,9$ & $-39,7$ \\
\hline Brasil & 112,08 & 100,10 & 44,08 & 29,51 & 17,07 & 1,1 & 2,9 & 2,5 & 7,1 & 22,3 & 1,1 & 0,9 & 0,5 & 0,4 & 0,2 & 98,2 & 93,1 & 92,4 & 65,9 & $-11,2$ \\
\hline EEUU & 117,58 & 109,30 & 134,40 & 124,37 & 149,28 & 4,5 & 15,6 & 16,9 & 22,1 & 23,4 & 13,7 & 13,2 & 12,3 & 10,1 & 12,9 & 90,9 & 67,0 & 67,9 & 58,0 & 61,1 \\
\hline México & 7,03 & 13,16 & 5,51 & 9,39 & 13,98 & 3,0 & 12,4 & 12,1 & 7,3 & 8,9 & 0,1 & 0,3 & 0,1 & 0,2 & 0,3 & 53,3 & $-5,7$ & $-30,7$ & 16,8 & 13,3 \\
\hline India & 20,91 & 34,62 & 31,67 & 11,01 & 11,26 & 0,0 & 0,4 & 2,1 & 8,3 & 21,8 & 0,1 & 0,2 & 0,3 & 0,1 & 0,2 & 99,9 & 98,3 & 83,6 & $-2,2$ & $-48,3$ \\
\hline España & 1842,19 & 1377,24 & 1705,59 & 1597,74 & 1627,61 & 29,8 & 96,6 & 113,1 & 122,7 & 118,8 & 32,8 & 25,4 & 32,5 & 27,6 & 27,5 & 96,5 & 85,7 & 85,3 & 83,0 & 84,8 \\
\hline Irán & 58,34 & 35,93 & 37,33 & 8,04 & 3,04 & 0,0 & 0,0 & 90,0 & 460,2 & 113,9 & 0,3 & 0,1 & 0,2 & 0,1 & 0,0 & 100,0 & 100,0 & 46,8 & $-91,0$ & $-87,3$ \\
\hline Egipto & 1141,89 & 2107,40 & 1690,67 & 4560,93 & 6801,60 & 0,8 & 0,1 & 0,3 & 0,6 & 0,8 & 0,9 & 1,4 & 1,9 & 7,7 & 9,9 & 99,4 & 100,0 & 99,9 & 100,0 & 99,9 \\
\hline Turquía & 284,70 & 356,79 & 322,89 & 427,03 & 474,98 & 13,9 & 0,5 & 27,4 & 21,6 & 16,1 & 1,2 & 1,7 & 2,1 & 3,3 & 4,0 & 85,8 & 99,2 & 80,6 & 84,5 & 90,0 \\
\hline Italia & 64,16 & 59,72 & 56,91 & 73,98 & 82,26 & 32,3 & 62,0 & 71,8 & 76,0 & 104,3 & 2,9 & 2,4 & 2,1 & 2,3 & 2,3 & 47,5 & 9,0 & $-2,5$ & 2,1 & $-7,6$ \\
\hline Sudáfrica & 1077,18 & 1358,92 & 1658,28 & 1992,28 & 2536,99 & 1,7 & 3,6 & 2,6 & 3,2 & 6,0 & 6,3 & 6,5 & 8,0 & 10,6 & 12,5 & 99,7 & 99,6 & 99,7 & 99,7 & 99,4 \\
\hline Marruecos & 4454,40 & 4229,44 & 3256,43 & 2516,65 & 1120,68 & - & - & - & 0,0 & 0,0 & 5,2 & 5,2 & 3,6 & 2,9 & 1,4 & - & - & - & 100,0 & 100,0 \\
\hline Pakistán & 1,01 & 13,47 & 3,12 & 12,13 & 15,71 & 0,1 & 0,0 & 0,1 & 0,3 & 1,9 & 0,0 & 0,0 & 0,0 & 0,0 & 0,0 & 90,1 & 100,0 & 76,5 & 56,0 & 34,9 \\
\hline Indonesia & 0,83 & 0,43 & 0,91 & 0,05 & 0,02 & 59,3 & 48,9 & 119,3 & 64,4 & 42,4 & 0,0 & 0,0 & 0,0 & 0,0 & 0,0 & $-96,0$ & $-96,8$ & $-97,7$ & $-99,8$ & $-99,9$ \\
\hline Japón & 0,00 & 0,01 & 0,04 & 0,02 & 0,05 & 129,8 & 110,9 & 83,3 & 62,0 & 61,5 & 0,0 & 0,0 & 0,0 & 0,0 & 0,0 & $-100,0$ & $-100,0$ & $-99,9$ & $-99,9$ & $-99,8$ \\
\hline Argentina & 321,31 & 309,89 & 321,35 & 342,59 & 149,48 & 5,6 & 46,7 & 2,8 & 5,2 & 3,8 & 1,2 & 1,4 & 1,2 & 1,5 & 0,6 & 96,7 & 75,8 & 99,2 & 97,8 & 96,1 \\
\hline Tailandia & 5,37 & 2,67 & 3,06 & 4,06 & 5,10 & 0,1 & 0,3 & 1,7 & 8,4 & 12,1 & 0,1 & 0,0 & 0,0 & 0,0 & 0,1 & 95,7 & 85,3 & 24,9 & $-25,5$ & $-46,5$ \\
\hline Túnez & 427,55 & 333,22 & 335,73 & 281,26 & 240,32 & 0,0 & 0,1 & 1,2 & 0,0 & 0,3 & 0,4 & 0,3 & 0,3 & 0,3 & 0,2 & 100,0 & 100,0 & 99,2 & 100,0 & 99,7 \\
\hline
\end{tabular}

